\title{
Green Synthesis of Silver Nanoparticles and Their Reduced Graphene Oxide Nanocomposites as Antibacterial Agents: A Bio-inspired Approach
}

\author{
Edreese Alsharaeh $^{1} \cdot$ Sarah Alazzam ${ }^{1} \cdot$ Faheem Ahmed $^{1}$. \\ Nishat Arshi ${ }^{1} \cdot$ Mohammed Al-Hindawi ${ }^{1}$ Garwin Kim Sing ${ }^{1}$
}

Received: 7 May 2016/Revised: 17 July 2016/Published online: 22 September 2016

(C) The Chinese Society for Metals and Springer-Verlag Berlin Heidelberg 2016

\begin{abstract}
In this paper, silver nanoparticles (AgNPs) and AgNPs/reduced graphene oxide (RGO) nanocomposites were prepared using lemon juice under microwave irradiation (MWI) and UV light irradiation. AgNPs with face-centered cubic structure RGO peaks were observed by X-ray diffraction. The UV-Vis spectrum showed modifications in the absorption peaks of the AgNPs with the concentration of the precursor solution and irradiation time, and the optimized condition was obtained for 20 min MWI and $60 \mathrm{~s}$ of UV light. Raman analysis confirmed the presence of RGO as D and G bands in the spectrum. Transmission electron microscopy analyses confirmed that the AgNPs of size ranging from 3 to $8 \mathrm{~nm}$ were anchored onto the RGO sheets. The antibacterial properties of the AgNPs/RGO nanocomposites were investigated using gram-negative bacteria. The results revealed that AgNPs/RGO nanocomposites consisting of approximately $5 \mathrm{wt} \% \mathrm{AgNPs}$ can achieve antibacterial performance similar to that of neat AgNPS. This method can be useful for the applications of AgNPs-based nanocomposites, where minute amount of silver will be utilized.
\end{abstract}

KEY WORDS: Silver nanoparticles (AgNPs); Nanocomposites; Reduced graphene oxide; Antibacterial

\section{Introduction}

There are increasing demands for finding new approaches to prevent and kill multidrug-resistant (MDR) strains of bacteria, as well as other MDR organisms [1]. Noble metal nanoparticles have been used as antibacterial agents since ancient times. Unlike antibiotics, bacteria cannot easily develop resistance to such nanoparticles, because these nanoparticles target multiple components in the bacterial cell [2-4]. Among noble metal nanomaterials, silver and gold nanoparticles (AgNPs and AuNPs) have distinctive features due to their attractive physicochemical properties

Available online at http://link.springer.com/journal/40195.

Edreese Alsharaeh

ealsharaeh@alfaisal.edu

1 College of Science \& General Studies, Alfaisal University, Riyadh, Saudi Arabia
$[5,6]$. Metal nanoparticles possess even more remarkable properties as a result of their small sizes and large surfaceto-volume ratios, which enable them to have more interactions with microorganisms, thus resulting in higher antibacterial efficacies [7-9]. Because of its unique properties in terms of mechanical strength, electrical conductivity and non-toxicity to mammalian cells, reduced graphene oxide (RGO) is an ideal substrate for tethering metal nanoparticles. As an allotrope of carbon, graphene consists of a single planar sheet of $s p^{2}$-bonded carbon atoms that are densely packed in a honeycomb crystal lattice. Some of the properties of graphene include a high surface area, good electrical conductivity, excellent biocompatibility and functionalization [10] and substantial mechanical strength. These properties attracted enormous interest for researchers to use graphene in the fields of physics, chemistry and biomedicine [11-13]. It has been reported that GO and other graphene derivatives can be used in biotechnology applications due to their effective interactions with biomolecules and relative non-toxicity to 
human cells. GO nanocomposites have attracted lot of interest as antibacterial agents, and AgNPs and RGO composites should exhibit the same antibacterial properties of free AgNPs. The advantages of this nanocomposite are as follows: first, it keeps the AgNPs well dispersed; when a suspension is composed of only AgNPs, the nanoparticles aggregate and precipitate. Moreover, it is cost effective because there will be less use of Ag. Additionally, the graphene will increase the surface area, thereby lowering the toxicity of the metal. Currently, the development of green chemistry approaches, which use biomaterials in the synthesis of various nanostructures has attracted a considerable amount of attention [14, 15].

In this work, we report the use of lemon as a biomaterial to synthesize AgNPs and AgNPs/RGO nanocomposites with variable sizes, and their antibacterial activities were tested toward a gram-negative bacteria. The results indicated that the prepared AgNPs/RGO nanocomposites, in which only $5 \mathrm{wt} \%$ AgNPs was used indeed possessed excellent antibacterial activity similar to that of neat AgNPs.

\section{Materials and Methods}

\subsection{Preparation of AgNPs}

In a typical synthesis, $45 \mathrm{~mL}$ of aqueous $\mathrm{AgNO}_{3}$ was prepared in different concentrations of 1,2 and $5 \mathrm{mM}$. Freshly squeezed lemon juice was centrifuged for $5 \mathrm{~min}$ (3000 rpm), the pulp and other impurities were extracted, and then the juice was centrifuged again until pure lemon extract was obtained. The pure lemon extract was filtered using a standard gravity filtration method to ensure its purity. Then, $5 \mathrm{~mL}$ of the lemon extract was added to each $\mathrm{AgNO}_{3}$ solution. The mixtures were kept in the dark for $2 \mathrm{~h}$. Then, each mixture was divided into two halves; the first half was kept under microwave irradiation (MWI) separately under a power of $100 \mathrm{~W}$, and samples were collected at time intervals of 40,60 and $80 \mathrm{~s}$. The second half of the $\mathrm{AgNO}_{3}$ and lemon solution was exposed to UV light with a wavelength of $365 \mathrm{~nm}$ for either $20 \min$ or $30 \mathrm{~min}$. The resulting solution turned a yellowish brown color, which is characteristic of AgNPs (see Fig. 1d).

\subsection{Preparation of Reduced Graphene Oxide (RGO)}

First, $400 \mathrm{mg}$ of GO [16] was suspended in $20 \mathrm{~mL}$ of deionized water and sonicated to produce a yellow homogeneous dispersion. Then, $400 \mu \mathrm{L}$ of hydrazine hydrate (HH; reducing agent) was added to the mixture and microwaved (KENWOOD; $740 \mathrm{~W}$ ) at $100 \%$ power for 30-s cycles (on for $10 \mathrm{~s}$ and stirring for $20 \mathrm{~s}$ ) for $2 \mathrm{~min}$. This process resulted in a gradual change in the color of the GO solution from yellow to black, indicating that the chemical reduction in GO was complete. The GO sheets were then pelleted by centrifugation (HERAEUS-LABOFUGE 400 Centrifuge, $5000 \mathrm{rpm}$ for $15 \mathrm{~min}$ ) and left to dry at $80{ }^{\circ} \mathrm{C}$ for $8 \mathrm{~h}$.

\subsection{Preparation of AgNPs/RGO Nanocomposites}

To prepare AgNPs/RGO nanocomposites, $5 \mathrm{wt} \%$ AgNPs in RGO was mixed in various molarities $(1,2$, and $5 \mathrm{mM})$, and $6 \mu \mathrm{L}$ of $\mathrm{HH}$ was added while stirring for $1 \mathrm{~min}$; then, the solution was sonicated for $60 \mathrm{~s}$. The solution was placed in the $\mathrm{MH}$ (CEM-Discover) for $10 \mathrm{~min}$ at the pressure of $150 \mathrm{psi}(1.034 \mathrm{MPa})$, and the temperature of $1500{ }^{\circ} \mathrm{C}$. After standard gravity filtration, the solution was dried for $8 \mathrm{~h}$ under vacuum at $60^{\circ} \mathrm{C}$.

\subsection{Characterization}

X-ray diffraction (XRD, Philips-PW 1729, Holland) patterns of the material were obtained using a $\mathrm{Cu}$ radiation source [30 kV, $40 \mathrm{~mA}, K_{\alpha}$ radiation $\left.(\lambda=0.154430 \mathrm{~nm})\right]$. Raman spectra were acquired using a Bruker Equinox 55 FTIR spectrometer equipped with an FRA106/S FT-Raman module and a liquid $\mathrm{N}_{2}$-cooled $\mathrm{Ge}$ detector using the 1064-nm line of a Nd:YAG laser with an output laser power of $200 \mathrm{~mW}$. Transmission electron microscopy (TEM) was performed using a JEOL JSM-2100F operating at $200 \mathrm{kV}$. For the TEM observations, a drop of the specimen dispersed in ethanol was placed on copper grids and dried. Room temperature optical absorption spectra were recorded in the range of $200-800 \mathrm{~nm}$ using a UV-Vis spectrophotometer (Agilent-8453).

\subsection{Antibacterial Testing}

The antibacterial activities of the AgNPs and AgNPs/RGO nanocomposites were studied against gram-negative bacterial strain Escherichia coli HB101 K12 (Biorad, Hercules, CA). The bacteria were cultured in Luria-Bertani (LB) nutrient broth at $37^{\circ} \mathrm{C}$ for $18 \mathrm{~h}$ and harvested while in the exponential log phase. One-hundred microliters of $E$. coli in the mid-log phase was evenly spread on LB culture medium plates. For dried samples, $100 \mathrm{mg}$ of the powder was placed in the center. For liquid samples, wells (diameter $=6 \mathrm{~mm}$ ) were punched in the agar plates and filled with the nanoparticle solutions $(100 \mathrm{mg})$, and the agar plates were incubated overnight at $37^{\circ} \mathrm{C}$ for $24 \mathrm{~h}$. 

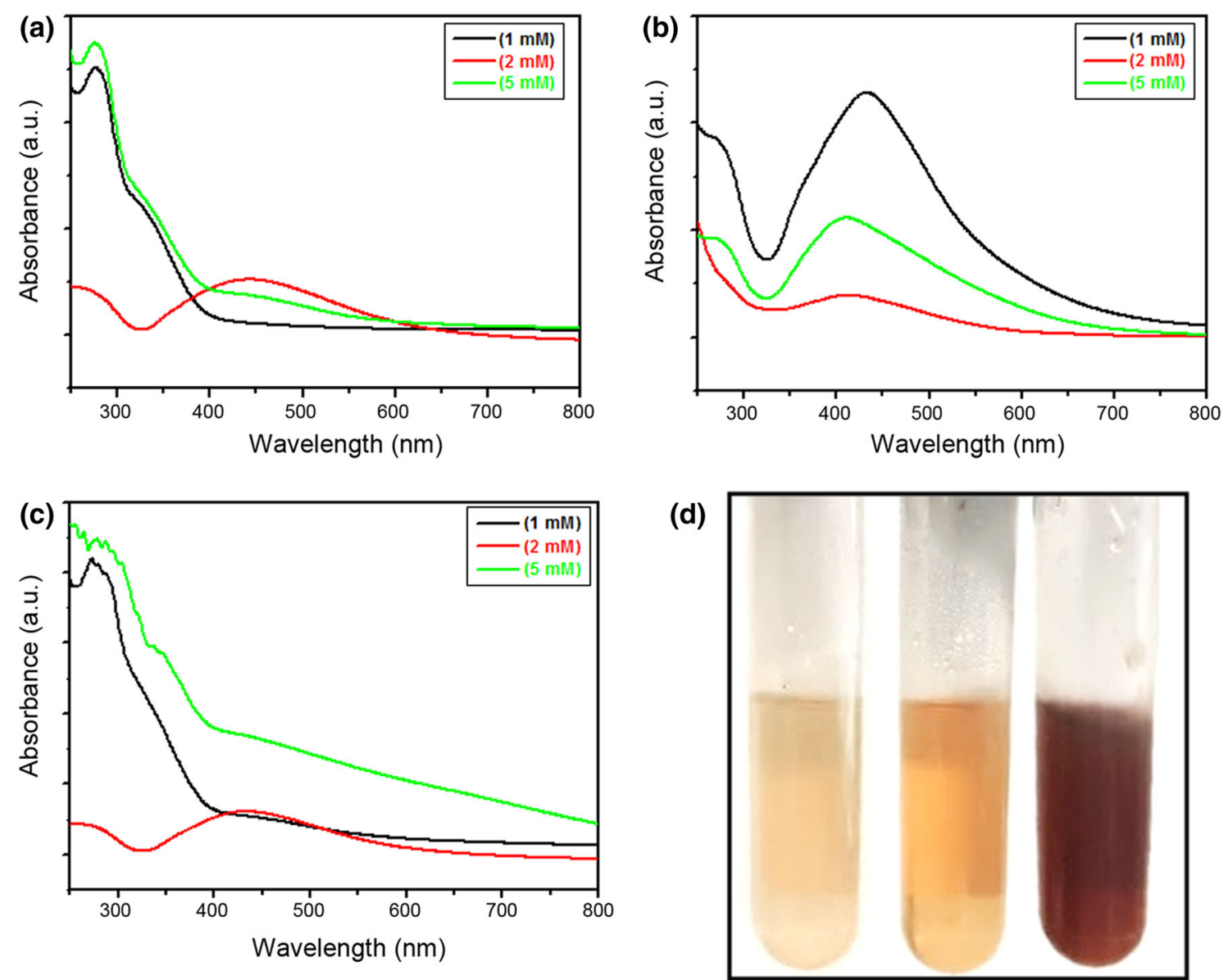

Fig. 1 UV-Vis spectra of AgNPs using lemon with various concentrations and processed using MWI times of: a $40 \mathrm{~s}$, b $60 \mathrm{~s}$, and c $80 \mathrm{~s}$. d Change in color of AgNPs formed by MWI for 40, 60 and $80 \mathrm{~s}$

\section{Results and Discussion}

Figure 1a-c presents the UV-Vis absorption spectra of lemon-synthesized AgNPs with various concentrations (1, 2 , and $5 \mathrm{mM}$ ) of silver nitrate under MWI for 40,60 and $80 \mathrm{~s}$. It can be clearly observed from the spectra that the absorption maxima occurred at approximately $420 \mathrm{~nm}$, indicating the formation of AgNPs. This prominent absorption maximum at $420 \mathrm{~nm}$ might be attributed to the Mie scattering by silver metal [17]. Interestingly, by increasing the concentration from 1 to $5 \mathrm{mM}$, modification in the intensity of the absorption peak was observed. Additionally, a more prominent effect of irradiation time was observed by increasing the irradiation time from 40 to $80 \mathrm{~s}$, as shown in Fig. 1a-c. The change of color of AgNPs is also shown in Fig. 1d.

Figure 2 presents the UV-Vis absorption spectra of lemon-synthesized AgNPs with various concentrations (1, 2 , and $5 \mathrm{mM}$ ) of silver nitrate under UV light irradiation. To observe the effect of UV light on the preparation of silver nanoparticles, the irradiation time was varied from
20 min (Fig. 2a) to 30 min (Fig. 2b). Both of these figures clearly show that the absorption maximum of the studied nanoparticles occurred at a position similar to the characteristic peak of AgNPs. The broadness of the peak is an excellent indicator of the size of the nanoparticles. As the particle size increases, the peak becomes narrower with the decrease in bandwidth and the increase in band intensity [18]. It has been reported that the absorption spectrum of spherical silver nanoparticles presents a maximum between 420 and $450 \mathrm{~nm}$ with a blue or red shift when the particle size decreases or increases, respectively. It was observed that both the solution concentration and the irradiation time affected the size of the nanoparticles, which resulted in the increase in peak intensity as the concentration and irradiation time increased.

Figure 3a presents the XRD patterns of AgNPs with various concentrations $(1,2$, and $5 \mathrm{mM})$ of silver nitrate. The XRD data show diffraction peaks at $2 \theta$ of $38.2^{\circ}$ and $44.4^{\circ}$, corresponding to the (111) and (200) planes of silver, respectively. Therefore, the XRD patterns confirmed the crystalline structure of the AgNPs. No peaks 

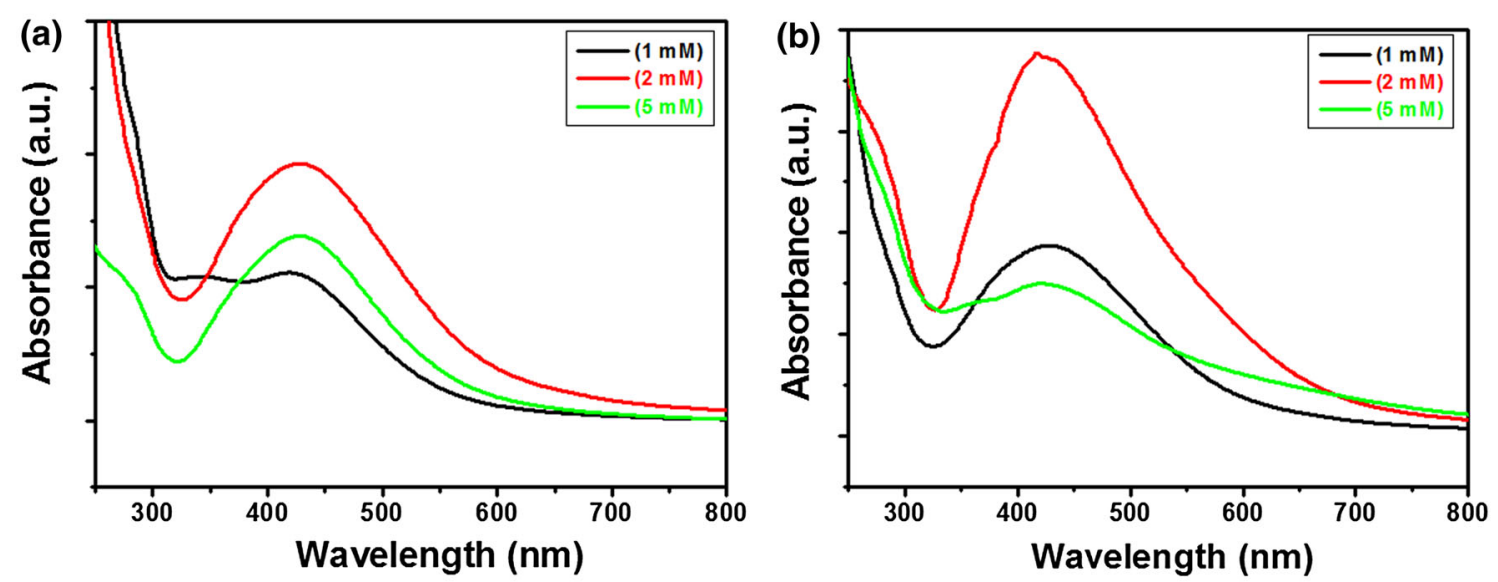

Fig. 2 UV-Vis spectra of AgNPs prepared using lemon with different concentrations and processed in UV light for: a 20 min, b 30 min
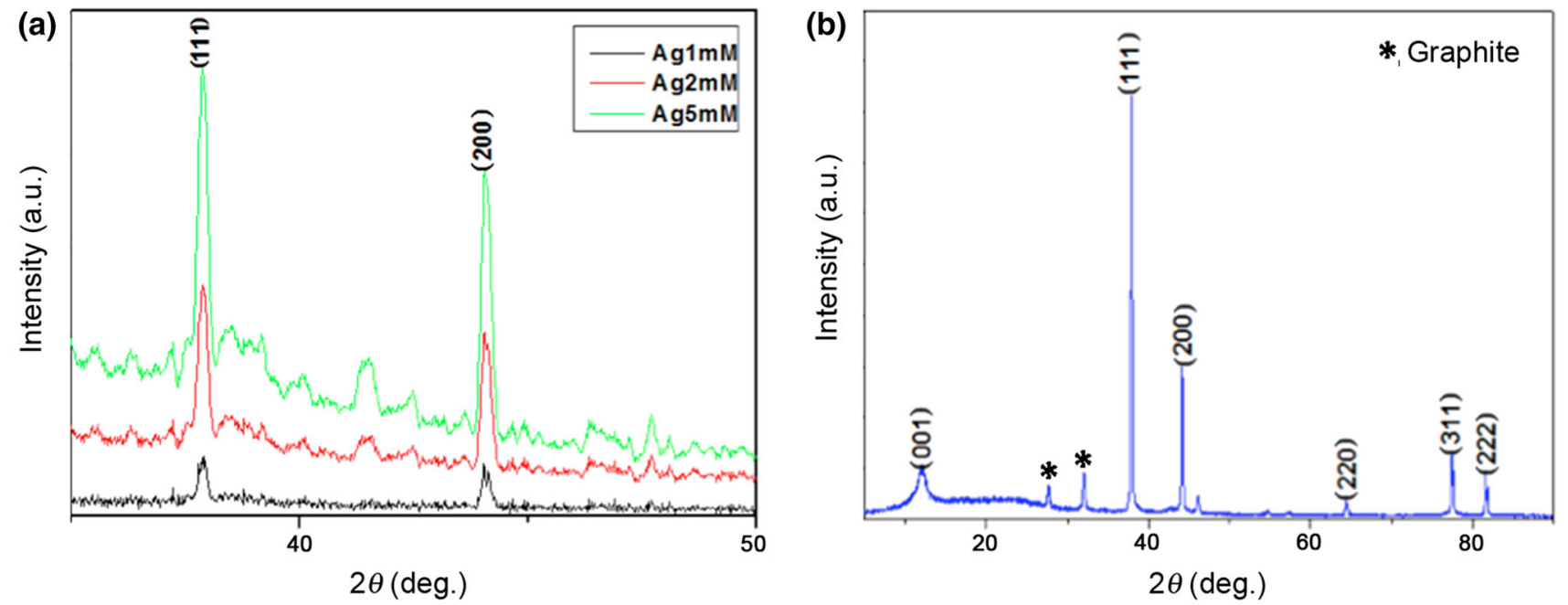

Fig. 3 XRD patterns of a AgNPs with different concentrations (1-5 mM) using MWI for $60 \mathrm{~s}$, b AgNPs/RGO nanocomposites prepared from $2 \mathrm{mM}$ under MWI for $60 \mathrm{~s}$

corresponding to other impurity crystalline phases were detected, and all the peaks in the XRD pattern can be readily indexed to the face-centered cubic (fcc) structure of Ag. From the patterns, it is clear that the intensity increases as the concentration increases, and the sample prepared using a concentration of $5 \mathrm{mM}$ has the highest peak intensity, whereas the sample prepared using a concentration of $1 \mathrm{mM}$ has the weakest peak intensity. The XRD pattern of the AgNPs/RGO nanocomposites prepared using MWI ( $2 \mathrm{mM}, 60 \mathrm{~s})$ is shown in Fig. 3b. It can be observed that GO presents a strong diffraction peak centered at $2 \theta$ of $10^{\circ}-13^{\circ}$, corresponding to the (001) interplane spacing. A broad diffraction peak can be observed at $2 \theta$ of $15^{\circ}-30^{\circ}$, which can be indexed to the disordered RGO sheets. The XRD patterns of the AgNPs/RGO nanocomposites exhibit characteristic peaks at $2 \theta$ of $38.42^{\circ}, 44.62^{\circ}, 64.72^{\circ}$ and $77.68^{\circ}$, which correspond to the (111), (200), (220) and (311) planes of fcc Ag, respectively, thus confirming the formation of AgNPs on RGO. In addition to these peaks, some low intensity peaks were also observed (marked with the asterisk) that might be due to the graphite, which was not reduced.

The TEM image of the AgNPs synthesized using lemon with $2 \mathrm{mM}$ of $\mathrm{AgNO}_{3}$ under MWI for $60 \mathrm{~s}$ is shown in Fig. 4a, while the TEM image of nanoparticles prepared with $2 \mathrm{mM} \mathrm{AgNO}_{3}$ under UV light irradiation for $20 \mathrm{~min}$ is shown in Fig. 4b. All of these images show that the nanoparticles are uniformly distributed and spherical in shape, with average diameters ranging from 3 to $8 \mathrm{~nm}$. These images indicate that the microwave synthesis provides monodisperse size distribution and that $2 \mathrm{mM} \mathrm{AgNO}_{3}$ with $60 \mathrm{~s}$ of MWI are the optimal conditions. This sample was subsequently used to prepare the nanocomposites.

The UV-Vis absorption spectra of the AgNPs/RGO nanocomposites prepared using the MWI (60 s, Fig. 5a) 

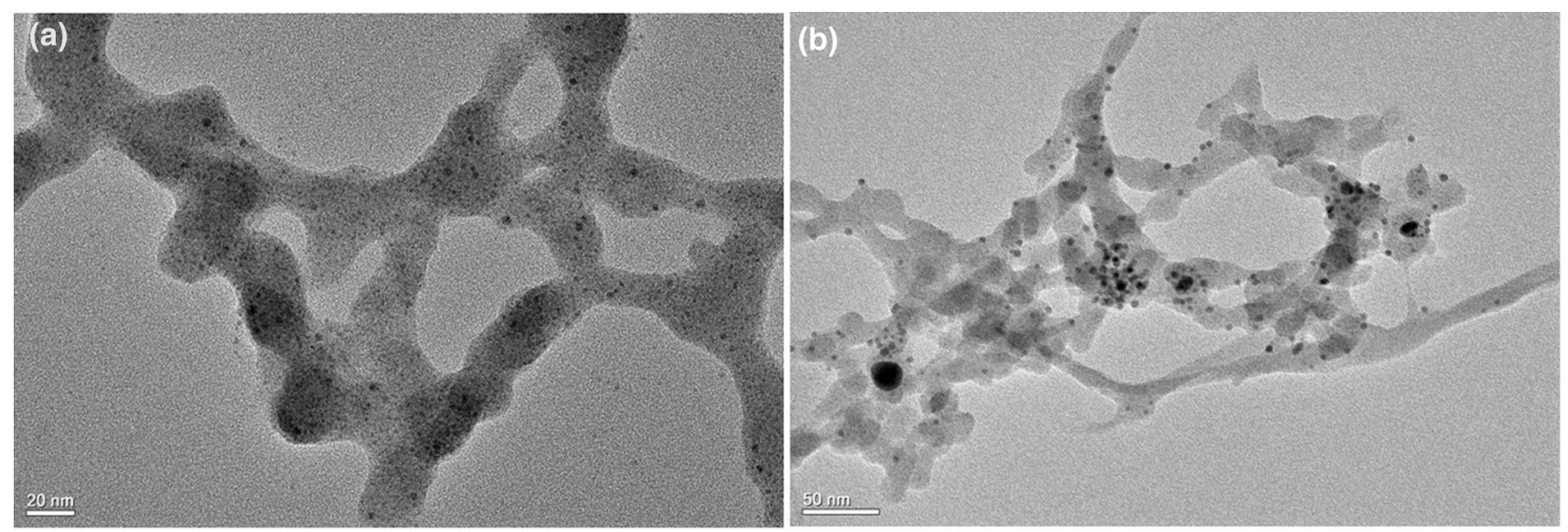

Fig. 4 TEM images of a AgNPs prepared from $2 \mathrm{mM}$ under MWI for $60 \mathrm{~s}$, b AgNPs prepared from $2 \mathrm{mM}$ under UV light for $20 \mathrm{~min}$
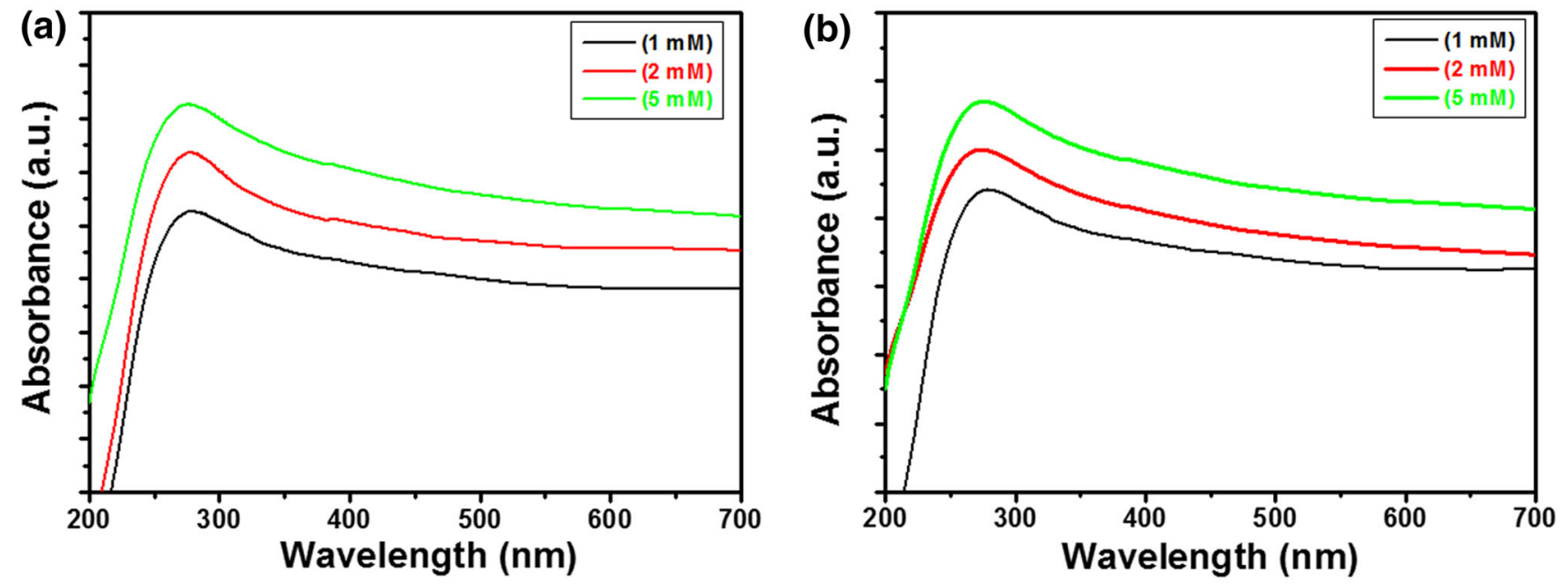

Fig. 5 UV-Vis spectra for AgNPs/RGO nanocomposites prepared using: a MWI for $60 \mathrm{~s}$, b UV light for 20 min

and UV light irradiation (20 min, Fig. 5b) methods with lemon and different concentrations of $\mathrm{AgNO}_{3}$ are shown in Fig. 5 for comparison. Both of the figures clearly show that the weak absorption maximum of the silver nanoparticles occurred at a position similar to that of the characteristic peak of AgNPs. In addition, the absorbance spectrum of the AgNPs/RGO nanocomposites presents an absorbance band at $274 \mathrm{~nm}$, which might be attributed to the characteristic absorption band of RGO. The broadness of the peak is an excellent indicator of the size of the nanoparticles. As the particle size increases, the peak becomes narrower with the decrease in the bandwidth and the increase in the band intensity [18]. The peak intensity was observed to increase as the concentration increased in both the methods.

The TEM image of the AgNPs/RGO nanocomposites synthesized using $2 \mathrm{mM} \mathrm{AgNO}_{3}$ with MWI for $60 \mathrm{~s}$ is shown in Fig. 6a. The TEM image of the nanocomposites prepared using $2 \mathrm{mM} \mathrm{AgNO} 3$ with UV light irradiation for $20 \mathrm{~min}$ is shown in Fig. 6b. These TEM images clearly indicate that the ideal conditions for synthesizing the composite are $2 \mathrm{mM} \mathrm{AgNO}_{3}$ with MWI for $60 \mathrm{~s}$. As shown in Fig. 6a, silver nanoparticles with $3-8 \mathrm{~nm}$ in size are uniformly dispersed on the surface of the well-ordered, dispersed RGO sheets, which effectively prevented the aggregation of silver nanoparticles. However, the nanocomposite prepared using UV light has defects in its RGO sheets: the sheets are crumbled, and the nanoparticles are agglomerated. Moreover, the TEM images clearly demonstrated that a relatively larger amount of AgNPs deposited on the surface of RGO for the samples prepared using $2 \mathrm{mM} \mathrm{AgNO}_{3}$ with MWI for $60 \mathrm{~s}$. All these results indicate the successful preparation of AgNPs/RGO nanocomposites. The presence of $\mathrm{Ag}$ in the elemental state in the AgNPs/RGO nanocomposite was confirmed by the EDX spectra shown in Fig. 6c.

Raman spectroscopy was used to characterize the nanostructure. Figure 7 presents the Raman spectrum of the AgNPs/RGO nanocomposite. The D and G bands are 

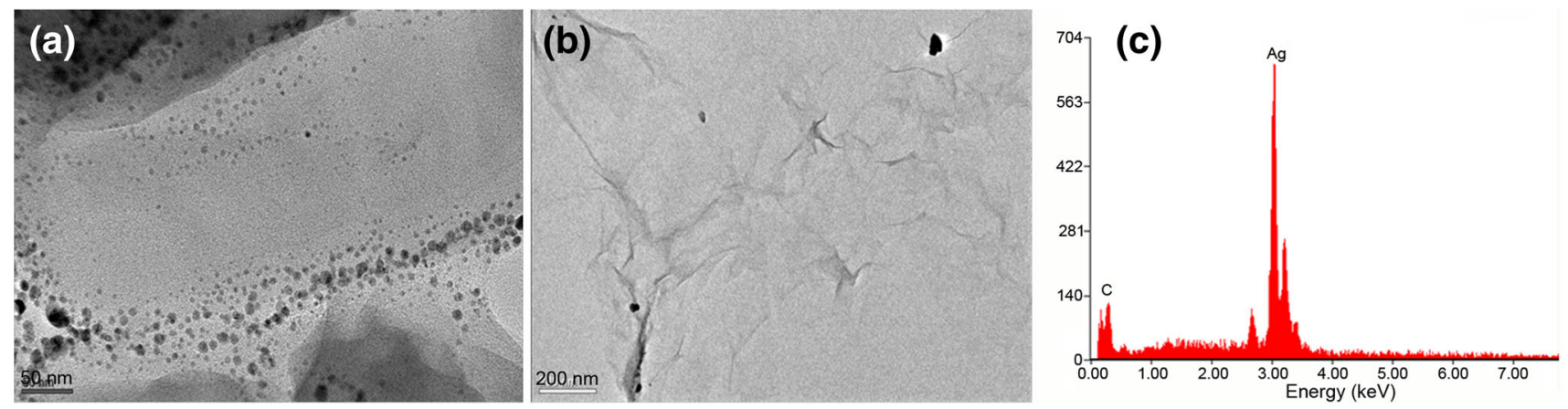

Fig. 6 TEM images of a AgNPs/RGO nanocomposites prepared from $2 \mathrm{mM}$ under MWI for $60 \mathrm{~s}$, b AgNPs/RGO prepared from $2 \mathrm{mM}$ under UV light for $20 \mathrm{~min}$ and $\mathbf{c}$ EDX spectrum of the AgNPs/RGO sample using a $2 \mathrm{mM}$ concentration under MWI for $60 \mathrm{~s}$

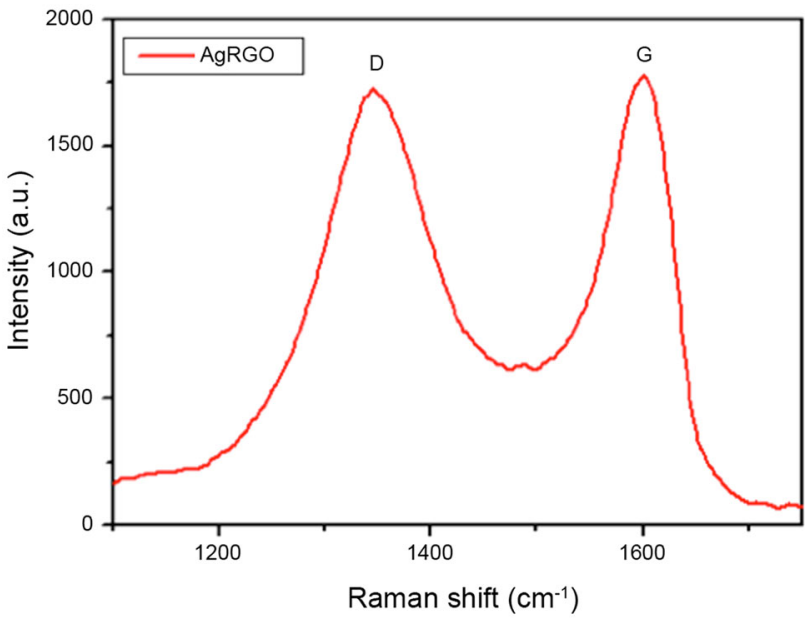

Fig. 7 Raman spectrum of AgNPs/RGO nanocomposites using a $2 \mathrm{mM}$ concentration under MWI for $60 \mathrm{~s}$

observed at approximately 1350 and $1600 \mathrm{~cm}^{-1}$, respectively. Sufficient information regarding the structure of graphene can be extracted from the Raman spectrum. The $\mathrm{G}$ band is a result of $s p^{2}$-bonded carbons present in graphene. The $\mathrm{D}$ band provides information regarding the breathing mode of the $\kappa$-point, and the $\mathrm{G}$ band is related to the tangential stretching mode of the $E_{2} g$ phonon of $s p^{2}$ carbon atoms [19]. The intensities of these two bands are high because of the attachment of AgNPs to the surface of RGO. The D/G intensity ratio was measured to be 0.98 . The intensity ratio of the $D / G$ bands is a measure of the defects present in the graphene structure.

The disk diffusion test was performed on reduced graphene oxide to investigate the antibacterial activity of RGO alone against E. coli. No inhibition zone for the E. coli bacteria was observed, as shown in Fig. 8a, which revealed that $E$. coli bacteria are unaffected by RGO alone. As shown in Fig. 8b-g, the AgNPs exhibit excellent antibacterial activity against $E$. coli. The disk diffusion assay results indicated that the samples prepared using $2 \mathrm{mM}$ $\mathrm{AgNO}_{3}$ with MWI for $60 \mathrm{~s}$ and $5 \mathrm{mM} \mathrm{AgNO}{ }_{3}$ with MWI for $60 \mathrm{~s}$ formed a 16-mm inhibition zone, which indicated slightly more antibacterial activity against gram-negative bacteria than the samples prepared under UV light. Moreover, the colony count assay showed that the viability test of the samples prepared using $1 \mathrm{mM} \mathrm{AgNO}_{3}$ with MWI for $60 \mathrm{~s}$ inhibited approximately $99 \%$ of bacterial growth, followed by the samples prepared using $5 \mathrm{mM}$ and $2 \mathrm{mM} \mathrm{AgNO}_{3}$, which inhibited bacterial growth by $3 \%$ and $5 \%$, respectively. The samples prepared under UV light also strongly inhibited bacterial growth, but the inhibition was slightly less than that of the samples produced under MWI.

Figure 9 shows the antibacterial activity of the AgNPs/ RGO nanocomposites. The AgNPs/RGO nanocomposites were tested for their antibacterial activity against $E$. coli bacteria; only $5 \mathrm{wt} \%$ AgNPs was used to create these nanocomposites. Additionally, this is the minimum amount of AgNPs required to make this nanocomposite antibacterial. By comparing the nanocomposites prepared using the two different methods of MWI and UV light irradiation, the nanocomposites prepared using MWI were found to be the superior antibacterial agent. Overall, the $2 \mathrm{mM}$ samples consistently provided the largest inhibition zone, whether they were synthesized using MWI or UV light. Therefore, the nanocomposites prepared using only $5 \mathrm{wt} \%$ AgNPs and RGO showed a similar inhibition zone to AgNPs alone; hence, these nanocomposites can be useful for future inexpensive and environmentally friendly antibacterial agents.

UV irradiation and MWI have been used to initiate the reduction by activating a sensitive agent. The results show that UV irradiation and MWI time affect the initial growth rate, as well as the saturation level; however, the $\mathrm{AgNO}_{3}$ concentration makes less effect. A mechanism can be proposed to understand these observations. One can assume that there is a certain number of sites on a unit area of the surface of citrate that could reduce $\mathrm{Ag}^{+}$ions upon UV photon absorption and by using MWI [20, 21]. With the increase in MWI and UV irradiation time, one should 


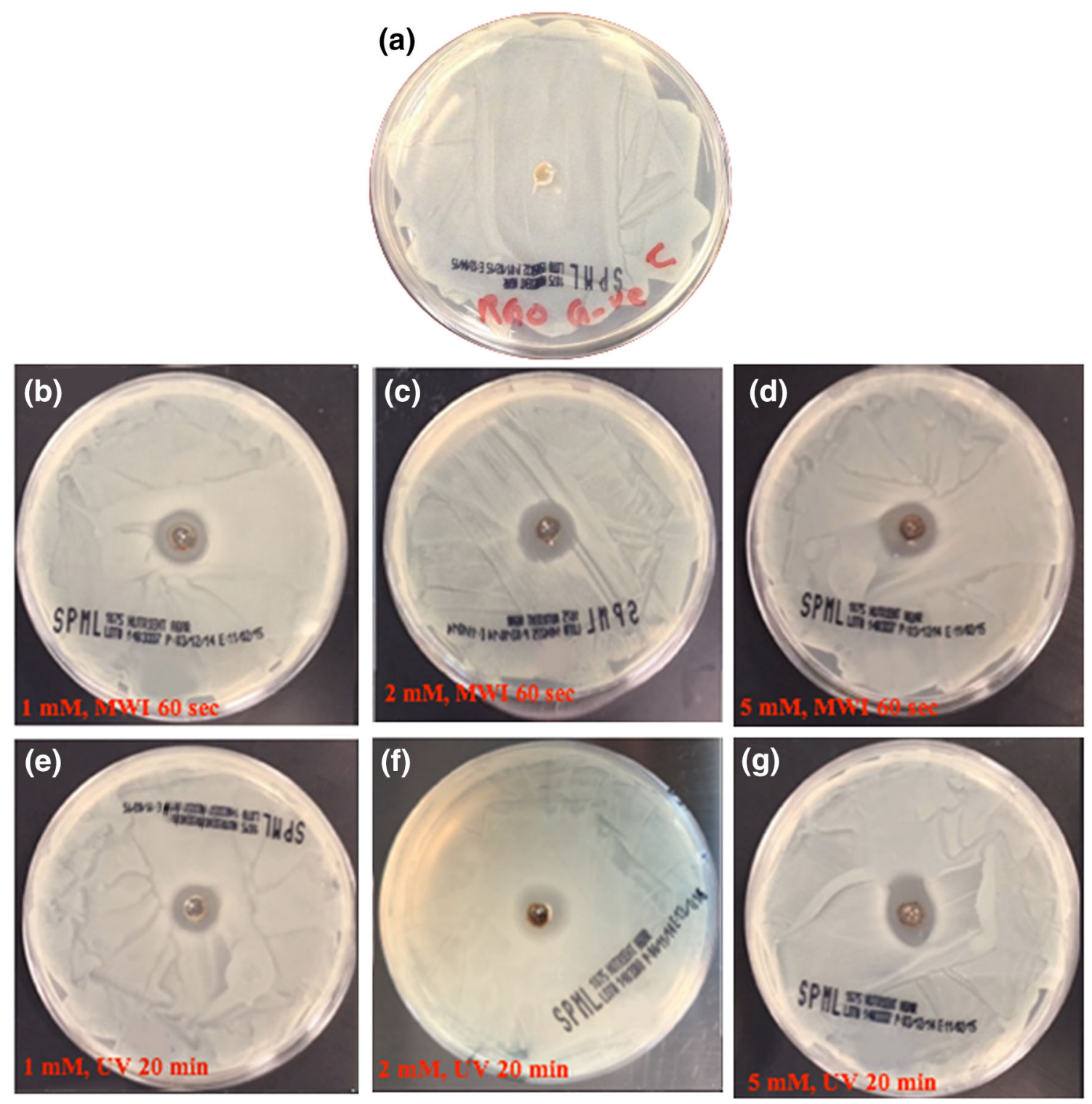

Fig. 8 Inhibition zones for a RGO alone, AgNPs for various concentrations (1-5 mM) using b-d MWI for $60 \mathrm{~s}$ and $\mathbf{e}-\mathbf{g}$ UV light irradiation for $20 \mathrm{~min}$

expect faster rate of $\mathrm{Ag}$ formation due to faster photon and microwave absorption. Interestingly, it was observed that the same amount of Ag is formed, if sufficient time is given (UV: $60 \mathrm{~s}$, MWI: $20 \mathrm{~min}$ ), regardless of UV intensity and MWI power.

The mechanism of the AgNPs/RGO nanocomposites formation can be explained as follows: before the reduction by citrate (lemon), the positively charged $\mathrm{Ag}^{+}$ions can be easily attracted to the negatively charged oxygen functional group on GO [22]. After the addition of lemon into the mixture, the lemon will release electron to reduce $\mathrm{Ag}^{+}$ions, and AgNPs can then be easily deposited on the RGO sheets due to the electrostatic attraction.

\section{Conclusion}

In summary, AgNPs and AgNPs/RGO nanocomposites have been successfully prepared using lemon as a biotemplate via the rapid and green MWI and UV light irradiation methods. XRD and Raman studies showed that the AgNPs and AgNPs/RGO nanocomposites possess an fcc structure, and UV-Vis measurements showed characteristic peak of Ag around $420 \mathrm{~nm}$. TEM images revealed that AgNPs with sizes ranging from 3 to $8 \mathrm{~nm}$ are anchored on the surface of the RGO nanosheets. The irradiation time of MWI and UV light played a key role in the formation of AgNPs with high Ag content, while the concentration of 

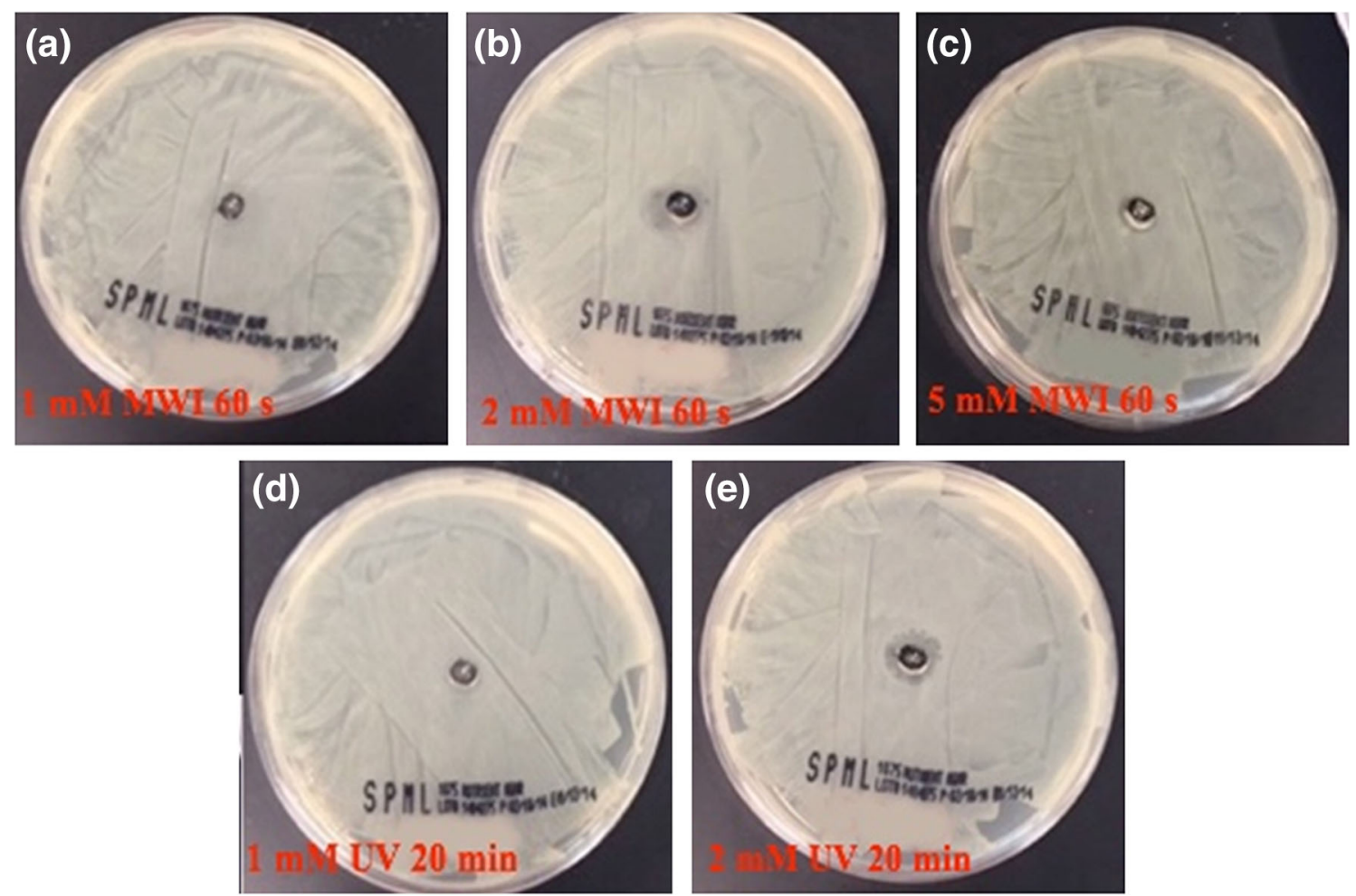

Fig. 9 Inhibition zones for AgNPs/RGO nanocomposites for various concentrations (1-5 mM) using a-c MWI for $60 \mathrm{~s}$ and d-e UV light irradiation for $20 \mathrm{~min}$

$\mathrm{AgNO}_{3}$ had less effect. The antibacterial activities of the AgNPs and AgNPs/RGO nanocomposites revealed that the inhibition zone of the $5 \mathrm{wt} \%$ AgNPs/RGO nanocomposites is almost identical to that of neat AgNPs. Meanwhile, the results showed that GO did not exhibit antibacterial activity. The synergistic effect between RGO and AgNPs has reduced the $\mathrm{Ag}$ content without compromising the antibacterial performance. The advantage of this AgNPs/ RGO nanocomposite with low Ag content will reduce the concern and risk of excessive Ag usage and provide a new direction for treating various diseases, which make it a potential material for effective antibacterial agents, food packaging and wound dressing applications.

Acknowledgment This work was supported by the Internal Research Grant, Alfaisal University (IRG 2014, No. 4050101011410).

\section{References}

[1] Y. Cui, Y. Zhao, Y. Tian, W. Zhang, X. Lu, X. Jiang, Biomaterials 33, 2327 (2012)

[2] J.L. Elechiguerra, J.L. Burt, J.R. Morones, A. Camacho-Bragado, X. Gao, H.H. Lara, M.J. Yacaman, J. Nanobiotechnol. 3, 6 (2005)

[3] O.V. Salata, J. Nanobiotechnol. 2, 3 (2004)

[4] I. Sondi, B. Salopek-Sondi, J. Colloid Interface Sci. 275, 177 (2004)
[5] I. Yacoby, I. Benhar, Nanomedicine 3, 329 (2008)

[6] J.S. Kim, E. Kuk, K.N. Yu, J.H. Kim, S.J. Park, H.J. Lee, S.H. Kim, Y.K. Park, Y.H. Park, C.Y. Hwang, Y.K. Kim, Y.S. Lee, D.H. Jeong, M.H. Cho, Nanomedicine 3, 95 (2007)

[7] W.S. Hummers, R.E. Offeman, J. Am. Chem. Soc. 80, 1339 (1958)

[8] L. Staudenmaier, Ber. Dtsch. Chem. Ges. 31, 1481 (1898)

[9] B.C. Brodie, Ann. Chim. Phys. 59, e472 (1860)

[10] C.N.R. Rao, A.K. Sood, K.S. Subrahmanyam, A. Govindaraj, Angew. Chem. Int. Ed. 48, 7752 (2009)

[11] Y. Wang, Z. Li, J. Wang, J. Li, Y. Lin, Trends Biotechnol. 29, 205 (2011)

[12] P. Avouris, C. Dimitrakopoulos, Mater. Today 15, 86 (2012)

[13] Z. Liu, J.T. Robinson, X. Sun, H. Dai, J. Am. Chem. Soc. 130, $10876(2008)$

[14] S. Mann, Biomimetic Materials Chemistry (Wiley, Hoboken, 1995)

[15] F.C. Meldrum, Int. Mater. Rev. 48, 187 (2003)

[16] E.H. Alsharaeh, A.A. Othman, Polym. Compos. 35, 2318 (2014)

[17] K. Aoki, J. Chen, N. Yang, H. Nagasawa, Langmuir 19, 9904 (2003)

[18] A.R. Shahverdi, A. Fakhimi, H.R. Shahverdi, S. Minaian, Nanomedicine 3, 168 (2007)

[19] F. Schedin, E. Lidorikis, A. Lombardo, V.G. Kravets, A.K. Geim, A.N. Grigorenko, K.S. Novoselov, A.C. Ferrari, ACS Nano 4, 5617 (2010)

[20] R.A. Stillings, R.J. Van Nostrand, J. Am. Chem. Soc. 66, 753 (1944)

[21] G.S. Egerton, J. Soc. Dyers Colour. 65, 764 (1949)

[22] C. Xu, X. Wang, Small 5, 2212 (2009) 